\title{
A Review Based Study of Diabetic Complications: Meta-Analysis in people with type 2 Diabetes Mellitus
}

\author{
*Nasser M.Al-Daghria,Mohammed Ghouse AhmedAnsaria, ShaunSabicoa, YousefAl-Salehab, Naji J.Aljohaniac, HananAlfawazad, \\ MohammedAlharbie.
}

Biomarkers of Osteoporosis, Biochemistry Department, College of Science, Saudi Arabia.

${ }^{*}$ Corresponding Author : Nasser M.Al-Daghria, Biomarkers of Osteoporosis, Biochemistry Department, College of Science

E-mail: ndaghri@ksu.edu.sa

Received date: April 01,2018;Accepted date : April 19,2018 ; Published date: April 29,2018.

Citation for this Article : Nasser M.Al-Daghria, A Review Based Study of Diabetic Complications: Meta-Analysis in people with type 2 Diabetes Mellitus. J. Endocrinology and Disorders Doi: 10.31579/2640-1045/016

Copyright : (c) 2018 Nasser M.Al-Daghria .This is an open-access article distributed under the terms of the Creative Commons Attribution License, which permits unrestricted use, distribution, and reproduction in any medium, provided the original author and source are credited.

\begin{abstract}
The prevalence of diabetes (DM) is constantly increasing worldwide at an alarming rate. According to the International Diabetes Federation in 2015, an estimated 415 million people globally were suffering from this condition. Complications of DM account for increased morbidity, disability, and mortality and represent a threat for the economies of all countries, especially the developing ones. The present special issue has been devoted to the recent progress in our understanding of diabetic complications, including the underlying molecular mechanisms, new diagnostic tools that facilitate early diagnosis, and novel treatment options.

This special issue focuses on progress and challenges in basic and clinical research on these chronic complications of diabetes. The end-stage consequences of diabetic complications can include severe vision loss; end-stage renal disease necessitating dialysis or transplant; myocardial infarction and stroke; and amputations. Many of these life-threatening or disabling events can be preventable with proper "life-long" diabetes care and a healthy lifestyle.
\end{abstract}

\section{Introduction}

The International Diabetes Federation estimates that 366 million people had diabetes in 2011, and that by 2030, this figure will have risen to a staggering 552 million worldwide. In 2011, diabetes was the cause of 4.6 million deaths and accounted for $11 \%$ of adult healthcare expenditure in the USA. The increasing incidence of both type 1 diabetes (T1D) and type 2 diabetes (T2D) elevates the complications of diabetes as one of the most important current public health issues. Complications of diabetes range from acute, life-threatening conditions such as severe hypoglycemia or ketoacidosis to chronic, debilitating complications affecting multiple organ systems, such as retinopathy, nephropathy, neuropathy, and cardiovascular disease. Estimates of the prevalence of diabetic complications are challenging, in part because there are no internationally agreed upon standards for diagnosis. However, a vast majority of those with diabetes will experience one of more of these complications of diabetes. For example, a recent analysis by the META-EYE study group reported that 93 million people worldwide suffer from diabetic retinopathy. For those with 20 or more years of diabetes, three quarters have some form of diabetic retinopathy.

\section{Diabetic Complications}

\section{a).Epidemiology and Pathogenesis of Diabetic Complications}

There is growing evidence that the underlying mechanisms in the pathogenesis of diabetic complications include certain genetic and epigenetic modifications, nutritional factors, and sedentary lifestyle. In a paper of this special issue entitled "Epigenetic Studies Point to DNA Replication/Repair Genes as a Basis for the Heritable Nature of Long Term Complications in Diabetes," A. A. Leontovich et al. using a zebrafish diabetic model, have explored the role of epigenetic mechanisms on the persistence of diabetic complications even after euglycemic control is achieved, a condition known as metabolic memory. They found that DNA-methylation, in or near genes belonging to the DNA replication/DNA metabolism process group, might play a key role in this process. Regarding basic risk factors for macro- and microvascular complications, the Irish Longitudinal Study on Ageing (TILDA), as M. L. Tracey et al. describe in their article "Risk Factors for Macro- and Microvascular Complications among Older Adults with Diagnosed Type 2 Diabetes: Findings from The Irish Longitudinal Study on Ageing," has recognized ageing, male gender, smoking, low level of physical activity, and high cholesterol as independent predictors of macrovascular complications. Conversely, smoking, hypertension, and duration of DM over 10 years proved to be predictive factors for microvascular complications.

(b) Microvascular Complications. Diabetic nephropathy, neuropathy, and retinopathy are the main microvascular complications induced by chronic hyperglycemia via several mechanisms such as the production of advanced glycation end products (AGEs), the creation of a proinflammatory microenvironment, and the induction of oxidative stress.

(c.)Macrovascular Complications. Atherosclerosis is more common in people with DM than in those without. For example, DM increases the risk for stroke in people aged 20 to 65 years more than 5 times. The present special issue includes articles on macrovascular complications of DM as well. J. Zhang et al. in the article entitled "Coronary Plaque Characteristics Assessed by 256-Slice Coronary CT Angiography and Association with High-Sensitivity C-Reactive Protein in Symptomatic Patients with Type 2 Diabetes" have performed a coronary Computed Tomography Angiography to evaluate coronary plaque subtypes and luminal narrowing in patients with and without type $2 \mathrm{DM}$. 
They report that patients with DM are more prone to have significant stenosis with calcified plaques and such findings are accompanied by higher hs-CRP levels. Moreover, in a review article entitled "The Role of AGE/RAGE Signaling in Diabetes-Mediated Vascular Calcification," A. M. Kay et al. emphasize the key role of AGE/RAGE signaling on the promotion of DM-mediated vascular calcification. In this process, many intracellular signaling pathways contribute to increased oxidative stress, which in turn leads to deposition of hydroxyapatite minerals into the extracellular matrix and vascular calcification.

(d)Miscellaneous Complications: Diabetic cardiomyopathy is a specific complication that develops independently of coronary artery disease or hypertension and it is possible to lead to increased morbidity and mortality . The aim of the study "Assessment of Left Ventricular Structural Remodelling in Patients with Diabetic Cardiomyopathy by Cardiovascular Magnetic Resonance" by Y. Shang et al. was to evaluate the structural remodeling of left ventricular (LV) mass in patients with diabetic cardiomyopathy (DCM) using cardiovascular magnetic resonance (CMR). The authors contend that CMR can be a valid tool to estimate LV remodeling and its severity in patients with DCM. Y. Yu et al. in their article entitled "The Protective Effect of Low Dose Ethanol on Myocardial Fibrosis through Downregulating the JNK Signaling Pathway in Diabetic Rats" have explored the protective role of low dose ethanol on myocardial fibrosis in diabetic rats. In this study, low dose ethanol consumption was associated with lower mean arterial pressure, lower heart rate, high hydroxyproline content, and collagen volume fraction in myocardial tissue, together with decreased expression of ALDH2 and downregulation of the JNK pathway. Finally, in the review paper "Molecular and Electrophysiological Mechanisms Underlying Cardiac Arrhythmogenesis in Diabetes Mellitus," G. Tse et al. discuss in detail the role of several cardiac factors (e.g., abnormalities in conduction or repolarization, electrophysiological, and structural remodeling) on arrhythmogenesis in patients with DM. They suggest that deeper investigation of these mechanisms can help towards defining new target molecules for potential future antiarrhythmic therapy for patients with DM.

\section{T2DM Complications}

Cardiovascular Disease: Cardiovascular disease is a primary cause of mortality and morbidity in both prediabetes and T2DM, the potential mechanism for which is oxidative stress that has important effects on atherogenesis and may contribute to low-density lipoprotein (LDL) oxidation. Prevention of premature cardiovascular events involves complex interactive treatments with antihypertensives, lipidlowering agents, and routine low-dose aspirin administration.

Diabetic Neuropathy: Diabetic neuropathy may be associated with foot ulcers, amputations, non-healing skin wounds, and sexual dysfunction. The neuropathy results in loss of protective sensation in the feet, which leads to callous formation, ulceration and other injury, and may also result in the infection of the skin (e.g. cellulitis) and/or bones of the foot (e.g. osteomyelitis) and gangrene. Sexual dysfunction usually occurs in young-aged diabetic patients because of oxidative stress in cavernous tissues.

Diabetic Nephropathy: Diabetic nephropathy is one of the most important microvascular complications, whose earliest manifestation is the presence of minute amounts of urinary protein (microalbumin) which cannot be detected in routine urinalysis, but is detectable by specific testing. If the detection can be done in the earlier phase, the progression of nephropathy can be prevented. This is, however, frequently overlooked because of the unawareness that the routine urinalysis lacks sensitivity in detecting micro albuminuria. .

Diabetic Retinopathy:The retina is the most vascular region in the body, as it needs high oxygen to convert light into electrical energy in the rods and cones. Chronic hyperglycemia may cause microvascular damage to the retinal vessels, resulting in edema and/or hemorrhage into the retina or the vitreous humor because of vascular permeability.
In fact, dysglycemia often occurs earlier than the diagnosis of diabetes patients, because nearly $20 \%$ of newly diagnosed diabetes patients show evidence of retinopathy

Cancers: Epidemiologic evidence has demonstrated that diabetes may elevate the risk of cancer such as colorectal cancer liver cancer, bladder cancer breast cancer, kidney cancer, which varies depending on the subsites of specific cancers. Mechanisms underlying the association of T2DM with cancer risk are as follows: firstly, T2DM and cancers usually share many risk factors such as age, obesity, sedentary lifestyle, smoking, higher intake of saturated fats and refined carbohydrates, and some psychology factors. Secondly, hyperinsulinemia is one of the major characteristics of T2DM. Meanwhile, it might promote carcinogenesis directly as it may promote the proliferation of colonic tumors in vitro and in experimental animals Besides, hyperinsulinemia may increase the level of IGF-1 which has mitogenic and antiapoptotic actions on cancer cells and the plasma or serum level of IGF-1 is also positively correlated with the risk of cancers.

New therapeutic strategies. Although oral antidiabetic agents and insulin are currently used for the treatment of T2DM and have brought about promising outcomes, problems still exist such as inadequate efficacy and adverse effects. We thus need to examine novel therapy strategies.

SGLT2 inhibitors: Sodium glucose co-transporter type 2 (SGLT2) inhibitors are a new class of glucose-lowering agents which prevent the reabsorption of renal-filtered glucose back into the circulation and increase urinary glucose elimination, thus lowering blood glucose levels They have been shown to be effective in reducing HbA1c, fasting plasma glucose (FPG), systolic blood pressure, bodyweight, as well as hyperglycaemia Dapagliflozin, one of the most advanced SGLT2 inhibitors, has been confirmed effective either as monotherapy or as addon therapy with metformin and insulin Adverse effects observed in the treatment of T2DM patients with dapagliflozin include genital infections and the occurrence of breast and bladder cancer . Long-term observational studies are, therefore, needed to examine possible negative effects.

DPP-4 inhibitors: Dipeptidyl peptidase-4 (DPP-4) inhibitors can improve the action of endogenous active GLP-1 and GIP by blocking its degradation by DPP-4 enzyme. They are effective in the protection of pancreatic $\beta$ cells and promotion of normal glucagon secretion, thus inhibiting the progression of T2DM. DPP-4 inhibitors are well tolerated because they play pivotal roles in cardiovascular protection and antiarteriosclerotic action, with few gastrointestinal side effects and weight neutrality. So far, available DPP-4 inhibitors include vildagliptin, sitagliptin, saxagliptin and linagliptin, which have been assessed in a variety of clinical studies about their pharmacokinetics /pharmacodynamics, safety, efficacy, and tolerabilty .Vildagliptin, one of the representative drugs, shows long-term advantages in the preservation of $\alpha$ - and $\beta$-cell functions, decrease in fasting lipolysis in adipose tissue, reduction in total cholesterol and lipotoxicity, and triglyceride storage in non-fat tissues such as muscle, pancreas and liver, with little drug interactions . Sitagliptin, another leading agent, available for use in Japan for the past few years, is now used in many T2DM patients with low insulin secretory capacity, whose efficacy and safety have been confirmed in many clinical practices

Lixisenatide: Lixisenatide (Lyxumia ${ }^{\circledR}$ ), a glucagon-like peptide (GLP)-1 receptor agonist, was approved for marketing by the European Medicines Agency in February 2013. Lixisenatide can activate the GLP-1 receptor, thus contributing to increasing insulin secretion, inhibition of glucagon secretion and decreasing gastrointestinal motility to promote satiety.In the GetGoal study program, HbA1c, FPG, and postprandial plasma glucose (PPG) were effectively decreased by the use of lixisenatide. Furthermore, lixisenatide reduced bodyweight and had therapeutic effects on glycemia when used as monotherapy or combined therapy with insulin and oral antidiabetic drugs. Nowadays, the primary combined therapy of lixisenatide seems to be with basal insulin, and clinical development as a combination product with insulin glargine (Lantus $\left.{ }^{\circledR}\right)$ is still undergoing. 
GPR40 agonists: G protein-coupled receptor 40 (GPR40) is a free fatty acid (FFA) receptor and Gq-type, Gq-coupled G protein-coupled receptor which is highly expressed in pancreatic $\beta$-cells The stimulation of GPR40 with FFAs leads to insulin secretion through $\beta$ cell-specific signaling pathway, which can be inhibited by the treatment with small interfering RNA. A large number of chemical compounds which can act as GPR40 agonists exhibit glucosedependent insulin secretion in vitro and in vivo, among which TAK875 can reduce fasting plasma glucose and $\mathrm{HbA} 1 \mathrm{c}$ levels in clinical trials Recently, Tanaka and his colleagues reported three novel GPR40 agonists AS2031477, AS1975063 and AS2034178, which could improve both acute glucose-dependent insulin secretion and chronic whole-body glucose metabolism. Among these GPR40 agonists, AS2034178 has been shown to reduce microvascular complications, thus it has a therapeutic potential to improve the prognosis of T2DM patients. In conclusion, GPR40 agonists represent a new class of drugs in the treatment of T2DM, especially AS2034178 is the most promising candidate.

Nitrate/Nitrite:Nitric oxide (NO) is a simple ubiquitous molecule which can play significant roles in almost every biological system. It is synthesized from L-arginine by NO synthase (NOS) enzymes including neuronal (nNOS), inducible (iNOS), endothelial (eNOS), and mitochondrial (mtNOS) NOS. In the body, nearly $90 \%$ of NO is converted to nitrate $\left(\mathrm{NO}_{3}^{-}\right)$, a stable end-product of NO. It has been demonstrated that $\mathrm{NO}_{3}{ }^{-}$and nitrite $\left(\mathrm{NO}_{2}{ }^{-}\right)$may have some therapeutic implications, such as decreasing blood pressure, reducing oxidative stress and reducing oxygen consumption during exercise. It is also demonstrated that inorganic nitrate therapy can reduce visceral fat accumulation, lower serum triglycerides and normalize a disturbed glucose tolerance in eNOS deficient mice These findings suggest the roles of $\mathrm{NO}_{3}{ }^{-}$and $\mathrm{NO}_{2}{ }^{-}$in the prevention and treatment of T2DM for reduced weight in long-term $\mathrm{NO}_{3}{ }^{-}$therapy. However, harmful effects exist in the $\mathrm{NO}_{3}{ }^{-} / \mathrm{NO}_{2}{ }^{-}$therapy: high levels of plasma $\mathrm{NO}_{3}{ }^{-}$(1) increase arterial pressure; (2) may cause early onset of hypertension; (3) increase incidence of diabetes; (4) induce kidney dysfunction; and (5) produce hypothyroidism. All in all, $\mathrm{NO}_{3}{ }^{-} / \mathrm{NO}_{2}{ }^{-}$shows potential roles in new therapeutic applications for human health, and also does potential human risks. $\mathrm{NO}_{3}{ }^{-} / \mathrm{NO}_{2}{ }^{-}$derived from natural sources such as vegetables may be one of the ideal choices. Further investigations are necessary in this field, especially in the identification of individuals who may benefit from the $\mathrm{NO}_{3}{ }^{-} / \mathrm{NO}_{2}{ }^{-}$therapy.

Stem cell educator therapy: Evidence has suggested that T2DM patients always display multiple immune dysfunctions and chronic metabolic inflammation. Researches have shown that monocytes/macrophages may be main players which contribute to these chronic inflammations and insulin resistance in T2DM patients.Stem cell educator therapy, a novel technology, is designed to control or reverse immune dysfunction. The procedure includes: collection of patients' blood circulating through a closed-loop system, purification of lymphocytes from the whole blood, co-culture of them with adherent cord blood-derived multi-potent stem cells (CB-SCs) in vitro and administration of the educated lymphocytes (but not the CBSCs) to the patient's circulation. Current phase I/ phase II studies suggest the safety and therapeutic efficacy of this kind of therapy in T2DM, with remarkably increased insulin sensitivities and notable improvement of metabolic control in T2DM patients. This new method exhibits great benefits in improving treatment and cure for T2DM, particularly in early-stage diabetic patients, which may help to cope with diabetes-associated complications and improve the quality of their life.

\section{Conclusion}

T2DM and its related complications impose heavy health burdens worldwide and there have been not effective measures to fully cope with the diseases. The main cause of the diabetes epidemic is the interaction between genetic and environmental risk. A number of other factors are also attributable to the diseases.
Whereas most antidiabetic agents have shown beneficial effects when used as monotherapy or combination therapy, they are also associated with negative effects, such as weight gain, hypoglycemia, gastrointestinal effects or cardiovascular disease. With increasing incidence of T2DM, searching an ideal therapy becomes one of the top priorities in combating this disease. To date, several therapeutic strategies have been developed, such as the use of SGLT2 inhibitors, DPP-4 inhibitors and GPR40 agonists. Above all, stem cell educator therapy opened avenues to develop new therapeutic strategies in the treatment of T2DM, with safety and high therapeutic efficacy. Further investigations should focus on (1) the exact mechanism contributing to T2DM and its related complications; (2) effective intervention trials and prevention measures to avoid the occurrence of this disease; (3) earlier diagnosis for earlier treatment; (4) novel drugs with more beneficial effects and less adverse effects, so as to conquer this disease and improve the quality of life and overall life span.

\section{References}

1. Tripathi BK, Srivastava AK. Diabetes mellitus: complications and therapeutics. Med Sci Monit. 2006;12(7):RA130-147.

2. Chen L, Magliano DJ, Zimmet PZ. The worldwide epidemiology of type 2 diabetes mellitus-present and future perspectives. Nat Rev Endocrinol. 2011;8(4):228-236.

3. Zhang PH, Chen ZW, Lv D. et al. Increased risk of cancer in patients with type 2 diabetes mellitus: a retrospective cohort study in China. BMC Public Health. 2012;12:567.

4. Gastaldelli A. Role of beta-cell dysfunction, ectopic fat accumulation and insulin resistance in the pathogenesis of type 2 diabetes mellitus. Diabetes Res Clin Pract. 2011;93(1):S60-65.

5. Ismail-Beigi F. Pathogenesis and glycemic management of type 2 diabetes mellitus: a physiological approach. Arch Iran Med. 2012;15(4):239-246.

6. Zhao Y, Jiang Z, Guo C. New hope for type 2 diabetics: Targeting insulin resistance through the immune modulation of stem cells. Autoimmun Rev. 2011;11:137-142.

7. Wild S, Roglic G, Green A. et al. Global prevalence of diabetes: estimate for the year 2000 and projections for 2030. Diabetes Care. 2004;127(5):1047-1053.

8. Diamond J. Medicine: diabetes in India. Nature. 2011;469:478-479.

9. Yang W, Lu J, Weng J. et al. Prevalence of diabetes among men and women in China. N Engl J Med. 2010;362:1090-1101.

10. Weigensberg MJ, Goran MI. Type 2 diabetes in children and adolescents. Lancet. 2009;373:1743-1744. 11. Poulsen P, Grunnet LG, Pilgaard K. et al. Increased risk of type 2 diabetes in elderly twins. Diabetes. 2009;58(6):1350-1355.

12. Sanghera DK, Blackett PR. Type 2 diabetes genetics: beyond GWAS. J Diabetes Metab. 2012;3(198):pii6948.

13. Poulsen P, Kyvik KO, Vaag A. et al. Heritability of type II (noninsulin- dependent) diabetes mellitus and abnormal glucose tolerance: a population-based twin study. Diabetologia. 1999;42:139-145.

14. Kobberling JTH. Emperical risk figures of first degree relatives of non-insulin dependent diabetes. London: Academic Press; 1982.

15. Sladek R, Rocheleau G, Rung J. et al. A genome-wide association study identifies novel risk loci for type 2 diabetes. Nature. 2007;445(7130):881-885.

16. Frayling TM, Timpson NJ, Weedon MN. et al. A common variant in the FTO gene is associated with body mass index and predisposes to childhood and adult obesity. Science. 2007;316(5826):889-894.

17. Scott LJ, Mohlke KL, Bonnycastle LL. et al. A genome-wide association study of type 2 diabetes in Finns detects multiple susceptibility variants. Science. 2007;316(5829):1341-1345.

18. Saxena R. et al. Genome-wide association analysis identifies loci for type 2 diabetes and triglyceride levels. Science. 2007;316(5829):1331-1336.

19. Zeggini E, Weedon MN, Lindgren CM. et al. Replication of genomewide association signals in UK samples reveals risk loci for type 2 diabetes. Science. 2007;316(5829):1336-1341.

20. Steinthorsdottir V, Thorleifsson G, Reynisdottir I. et al. A variant in CDKAL1 influences insulin response and risk of type 2 diabetes. Nat Genet. 2007;39(6):770-775. 
21. Wellcome Trust Case Control Consortium. Genome-wide association study of 14,000 cases of seven common diseases and 3,000 shared controls. Nature. 2007;447(7145):661-678. [

22. van Exel E, Gussekloo J, de Craen AJ. et al. Low production capacity of interleukin-10 associates with the metabolic syndrome and type 2 diabetes: the Leiden 85-Plus Study. Diabetes. 2002;51(4):1088-1092.

23. Hua Y, Shen J, Song Y. et al. Interleukin-10-592C/A, $-819 \mathrm{C} / \mathrm{T}$ and $-1082 \mathrm{~A} / \mathrm{G}$ polymorphisms with risk of type 2 diabetes mellitus: A HuGE review and meta-analysis. PLoS One. 2013;8(6):e66568.

24. Unoki H, Takahashi A, Kawaguchi T. et al. SNPs in KCNQ1 are associated with susceptibility to type 2 diabetes in East Asian and European populations. Nat Genet. 2008;40(9):1098-1102.
25. Tsai FJ, Yang CF, Chen CC. et al. A genome-wide association study identifies susceptibility variants for type 2 diabetes in Han Chinese. PLoS Genet. 2010;6(2):e1000847.

26. Voight BF, Scott LJ, Steinthorsdottir V. et al. Twelve type 2 diabetes susceptibility loci identified through large-scale association analysis. Nat Genet. 2010;42(7):579-589.

27. Yasuda K, Miyake K, Horikawa Y. et al. Variants in KCNQ1 is associated with susceptibility to type 2 diabetes mellitus. Nat Genet. 2008;40(9):1092-1097. 